\title{
Erratum to: Analysis of peri-islet CD45-positive leucocytic infiltrates in long-standing type 1 diabetic patients
}

\author{
Shiva Reddy ${ }^{1}$ - Nina Zeng ${ }^{1} \cdot$ Hussam Al-Diery ${ }^{1} \cdot$ Doran Jung $^{1} \cdot$ Clifton Yeu $^{1}$ • \\ Maximilian O. Joret ${ }^{1} \cdot$ Mervyn J. Merrilees ${ }^{2} \cdot$ Fiona $\mathrm{Wu}^{3}$
}

Published online: 21 May 2015

(C) Springer-Verlag Berlin Heidelberg 2015

\section{Erratum to: Diabetologia}

DOI 10.1007/s00125-015-3519-6

Unfortunately, two entries were incorrect in Table 1. For case 6052 the correct ethnicity of the donor is African-American and not White and the C-peptide value is $0.06 \mathrm{nmol} / \mathrm{l}$ and not $<0.02 \mathrm{nmol} / \mathrm{l}$.

The authors are confident that these errors do not compromise the study design, analysis, interpretation or conclusions.

In the Acknowledgements it was incorrectly stated that M. Campbell-Thompson had left nPOD. The sentence should have read: 'We are grateful to $\mathrm{nPOD}$ for supplying pancreatic sections for this study and to M. Campbell-Thompson (nPOD) and S. Richardson (Peninsula School of Medicine, Plymouth, UK) for advice regarding antibodies and protocols.' Dr Richardson is currently based at the Institute of Biomedical and Clinical Sciences, University of Exeter, Exeter, UK.

The online version of the original article can be found at http://dx.doi.org/ 10.1007/s00125-015-3519-6.

Shiva Reddy

s.reddy@auckland.ac.nz

1 Department of Molecular Medicine and Pathology, Faculty of Medical and Health Sciences, University of Auckland, Private Bag 92019, Auckland, New Zealand

2 Department of Anatomy with Radiology, Faculty of Medical and Health Sciences, University of Auckland, Auckland, New Zealand

3 Diabetes Unit, Auckland District Health Board, Auckland, New Zealand 